\title{
Serotonin levels and 1-year mortality in patients with neuroendocrine tumors: a systematic review and meta-analysis
}

\author{
Vijay N Joish*,1, Sandip Shah², Jonothan C Tierce², Deep Patel², Chad McKee ${ }^{1}$, Pablo \\ Lapuerta $^{1}$ \& Jerome Zacks ${ }^{3}$ \\ ${ }^{1}$ Lexicon Pharmaceuticals, Inc., The Woodlands, TX 77381, USA \\ ${ }^{2}$ MKTXS, Raritan, NJ 08869, USA \\ ${ }^{3}$ Icahn School of Medicine, Center for Carcinoid and Neuroendocrine Tumors, Mount Sinai Health System, New York, NY \\ 10029, USA \\ *Author for correspondence: Tel.: +908 360 4782; vjoish@lexpharma.com
}

\begin{abstract}
Aim: Elevated serotonin in patients with neuroendocrine tumors (NETs) may impact heart failure incidence but a quantitative relationship has not been established. Materials \& methods: Systematic review and meta-analysis of studies assessing 24-h urinary 5-hydroxyindoleacetic acid (u5-HIAA) and mortality in patients with NETs (2007-2017) with a primary outcome of 1-year mortality risk and 24-h u5-HIAA. Results: We identified 1715 records of which 12 studies including 755 patients (3442 person-years with 376 deaths) were eligible for meta-analysis. Mean u5-HIAA was $149.2 \mathrm{mg} / 24 \mathrm{~h}$ (standard deviation: 96.6 ) and mortality was $13.0 \%$. The meta-regression equation showed an $11.8 \%\left(95 \% \mathrm{Cl}: 8.9-17.0 \% ; l^{2}=93.0 \%\right)$ increase in 1-year mortality for every ten-unit increase in u5-HIAA. Conclusion: Serotonin measured by its metabolite u5-HIAA is predictive of 1-year all-cause mortality in patients with NETs.
\end{abstract}

First draft submitted: 27 December 2018; Accepted for publication: 29 January 2019; Published online: 8 February 2019

Keywords: carcinoid syndrome $\bullet$ mortality $\bullet$ neuroendocrine tumors $\bullet$ serotonin

Neuroendocrine tumors (NETs) are relatively rare, with an incidence of approximately 70 cases per 1 million people, but present significant challenges in detection and management [1-4]. The prevalence of NETs has increased steadily from 0.006 to $0.048 \%$ over the past 20 years due to improvements in recognition, characterization, imaging and management $[1,5-7]$. In some patients, a NET can metastasize to the liver and drive the over-secretion of peptides, neuroamines and hormones such as serotonin and histamine [8,9]. Most NETs are considered secretory, or functional, tumors, a certain percentage of which can lead to the development of carcinoid syndrome (CS) [9]. CS is typically characterized by flushing and diarrhea but can also include dyspnea, wheeze and heart valve damage [10,11]. In the latter case, if serotonin levels are chronically elevated, carcinoid heart disease (CaHD) may develop [12]. CaHD has an estimated incidence of five cases per 1 million people; as many as 70\% of patients with CS may develop CaHD during the course of their disease [13,14].

$\mathrm{CaHD}$ is driven by high serotonin levels [12]. This elevated serotonin facilitates the formation of endocardial fibrotic plaques by stimulating fibroblast proliferation, primarily on the right side of the heart [12]. CaHD can be difficult to diagnose and may not be detected until symptoms of right heart failure emerge, such as shortness of breath on exertion, fatigue and ankle edema [12]. The poor cardiac status of patients with CaHD negatively impacts patients' quality of life and increases risk of mortality [15]. Among patients with small intestine NETs, a 5-year overall survival rate of $37 \%$ has been reported for those with CaHD, compared with $71 \%$ for those without CaHD [16]. Median survival for NET patients with CaHD has been reported to range from 1.6 to 4.6 years [14].

High systemic serotonin has been implicated in CaHD [12]. While an elevated serotonin level is considered a strong predictor of heart disease progression, a precise correlation between its metabolic product, 5-hydroxyindoleacetic acid (5-HIAA), and survival in patients with NETs has remained elusive [17,18]. For example, Zandee et al. did not find urinary 5-HIAA (u5-HIAA) to predict clinical outcome when adjusted for disease severity and other biomarkers, while Tirosh et al. have reported 5-HIAA to be useful for risk stratification and disease progression

Future $\because$ Medicine 
in patients with NETs $[19,20]$. To further explore this relationship, we conducted a systematic literature review and meta-analysis of studies investigating associations between 5-HIAA and mortality in patients with NETs.

\section{Materials \& methods}

\section{Search strategy \& selection criteria}

We conducted a systematic literature review and meta-analysis of summary data from randomized controlled trials or observational studies reporting u5-HIAA and mortality outcomes in patients with NETs. We followed the PRISMA (www.prisma-statement.org) guidelines for reporting of systematic reviews and meta-analyses. Studies had to be published in English between 1 January 2007 and 31 December 2017 and indexed in MEDLINE ${ }^{\circledR} /$ PubMed $^{\circledR}$ or Embase ${ }^{\circledR}$ databases. Studies that did not report a mortality outcome in patients with NETs, CS or CaHD, or reported outcomes as categorical or otherwise incompatible measures, were excluded, as were case reports, editorials, letters and other publications not reporting original outcomes. Search terms included ['neuroendocrine tumor' OR 'carcinoid syndrome' OR 'carcinoid heart disease' OR 'gastroenteropancreatic tumor' OR 'GEP NET' OR 'midgut neuroendocrine tumor' OR 'midgut NET' OR 'ileal neuroendocrine tumor' OR 'ileal NET' OR 'small bowel neuroendocrine tumor' OR 'small bowel NET'] AND ['urinary 5-HIAA' OR '5-hydroxyindoleacetic acid'] AND ['mortality' OR 'death']. Publishers or authors were contacted to request copies of records not readily available for purchase or otherwise. The primary search was supplemented by a hand search of grey literature sources and bibliographies of published studies including previously published reviews, Google Scholar (https: //scholar.google.com), and studies registered with ClinicalTrials.gov (https://clinicaltrials.gov). Study screening and data extraction were performed by two independent reviewers (D Patel, J Verma) with adjudication by a third reviewer (HD Shao).

\section{Data analysis}

Data were extracted into table shells defined a priori including study, patient and outcome information (study design, year of publication, corresponding author, number of patients, study period, duration of follow-up, explicit presence of CS or CaHD, mean 24-h u5-HIAA and number of deaths), and checked for redundancy by two independent extractors. Data extraction and risk of bias assessments were performed according to standards set forth by the Cochrane Handbook for Systematic Reviews of Interventions [21]. Heterogeneity was assessed using the $I^{2}$ statistic.

We constructed a statistical model to explore the relationship between u5-HIAA and mortality in NET patients. The hypothesis was that 1-year mortality would be higher with increasing levels of 5-HIAA. Initial Pearson correlation between u5-HIAA and mortality yielded a coefficient of 0.48 which supported further regression analysis. Shapiro-Wilk and Anderson-Darling tests indicated non-normality of the dataset, which was then logtransformed using the R logit function. Mortality outcomes were all converted to person-years for pooled analysis of included studies.

A random effects meta-regression model using the DerSimonian and Laird approach and the restricted maximum likelihood method (REML) was used to estimate the relationship between 5-HIAA levels and 1-year mortality [22]. Mortality event rate was based on the number of patient deaths during the reported study period divided by the total number of patients in the study. All mortality outcomes were converted to 1-year estimates and all 5-HIAA values were converted to mg per $24 \mathrm{~h}$.

All authors reviewed the selected studies for appropriateness according to the inclusion criteria and analysis plan, and all authors had access to and reviewed the extracted study data. All analyses and statistical modeling were conducted using $\mathrm{R}$ software, including the metafor, meta, weights, metagen and plotly packages [23-27].

\section{Role of the funding source}

The funder of the study, Lexicon Pharmaceuticals, Inc. (The Woodlands, TX, USA), participated in study design, data interpretation and writing of the report. The corresponding author had full access to all the data in the study and had final responsibility for the decision to submit for publication.

\section{Results}

A total of 1715 records were screened by title and abstract of which 208 qualified for full-text review. Twelve studies reporting 24-h u5-HIAA and mortality in 757 patients contributed a total of 3442 person-years that were included in the analysis (Figure 1). Individual study arms were distinguished from two trials [28,29], for a total of 14 


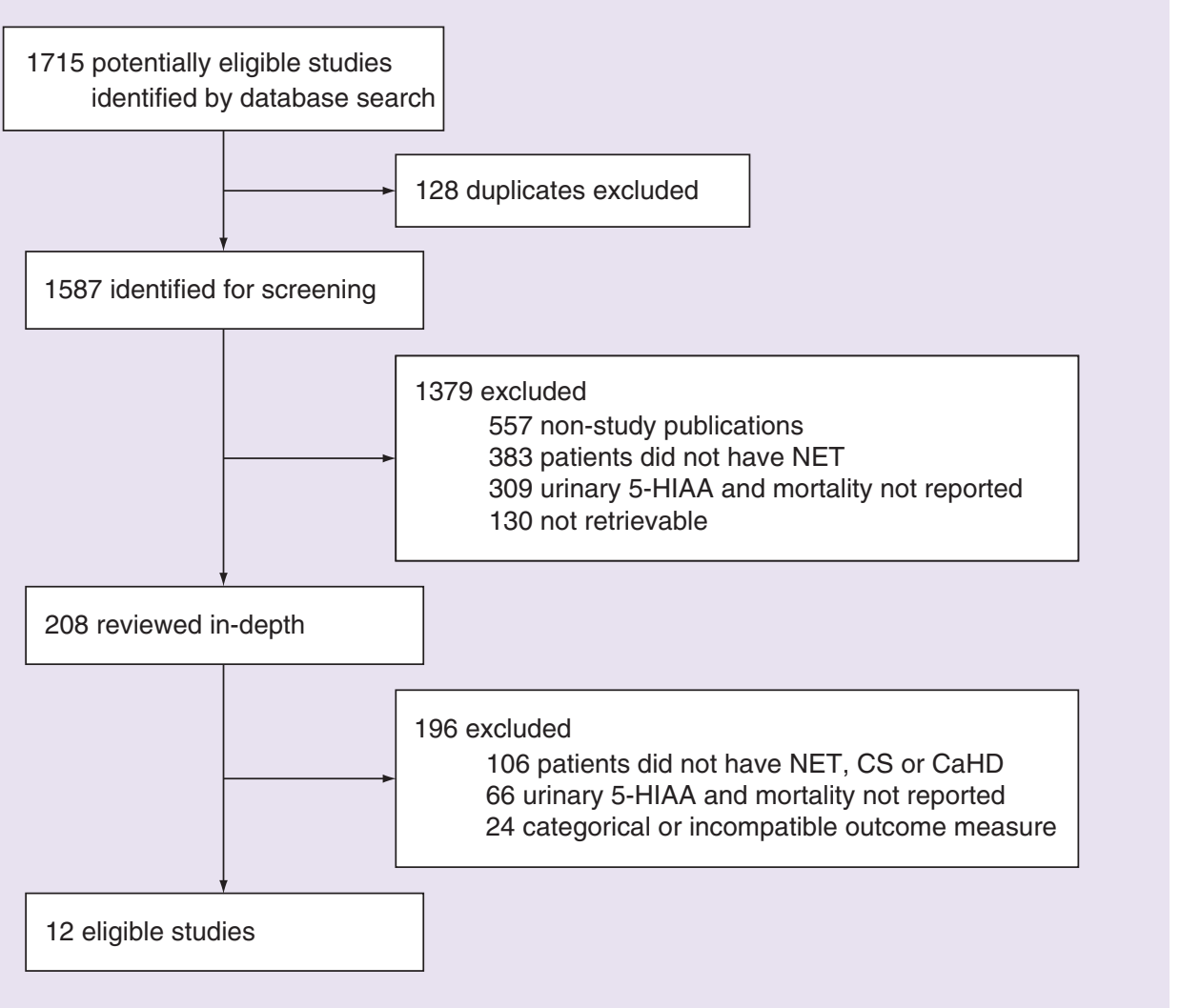

Figure 1. Study selection.

5-HIAA: 5-hydroxyindoleacetic acid; CaHD: Carcinoid heart disease; CS: Carcinoid syndrome; NET: Neuroendocrine tumor.

analyzable study arms from the 12 studies. Eight of the 14 study arms included patients with NETs and CaHD, and two with NETs and CS, specifically. Characteristics of the included studies are presented in Table 1.

Mean u5-HIAA across all studies was $149.2 \mathrm{mg} / 24 \mathrm{~h}$ (standard deviation: 96.6). The reference range for this marker is 3.0 to $15.0 \mathrm{mg} / 24 \mathrm{~h}$ [40]. The overall mortality rate was $13.0 \%$ (95\% CI: 9.0-20.0\%; Figure 2). Study arms comprized of patients with a NET and CS specifically had average 5-HIAA levels of 161.0 (two studies) with a mortality rate of $8.2 \%$. Those with a NET and CaHD had average 5-HIAA levels of 199.2 (eight studies) with a mortality rate of $27.8 \%$.

Every ten-unit increase in u5-HIAA predicted an $11.8 \%$ (95\% CI: 8.9-17.0\%) increase in 1-year mortality (Figure 3). The association between higher 5-HIAA and mortality remained significant after controlling for underlying comorbidity (NET only, CS, CaHD; $\mathrm{p}=0.007$ ). The heterogeneity among studies was expectedly high $\left(I^{2}=93 \%\right)$. The risk of bias assessment revealed no significant sources of bias among studies included in the meta-analysis (Supplementary Data).

\section{Discussion}

This systematic review and meta-analysis demonstrated a significant relationship between elevated u5-HIAA and mortality in patients with NETs. Every ten-unit increase in u5-HIAA predicted an $11.8 \%$ increase in 1-year mortality among this vulnerable population. Previous reports have correlated chronic elevated serotonin levels with $\mathrm{CaHD}$ and poorer outcomes [12,41,42]. The relationship between urinary levels of its metabolite, 5-HIAA and long-term outcomes in patients with NETs has not been clearly defined. Though several informal narrative reviews are available, to our knowledge this is the first systematic literature review and quantitative analysis closely examining reports of $\mathrm{u} 5$-HIAA and mortality in this population.

NETs that lead to CaHD are secretory in nature. The vasoactive substances released by secretory NETs, including 5-hydroxytryptamine (serotonin; 5-HT), histamine, tachykinins and prostaglandins are metabolized by the liver and 


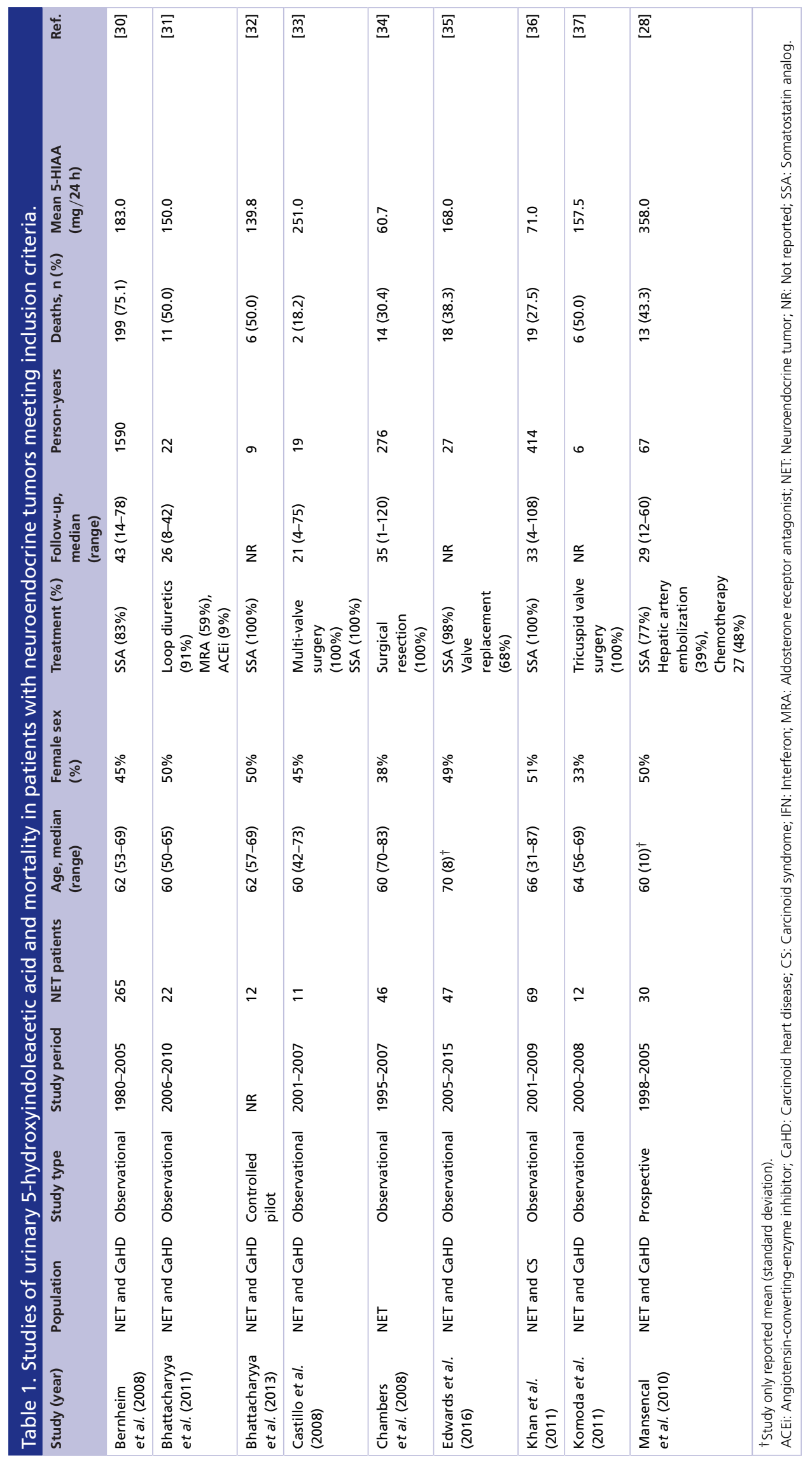




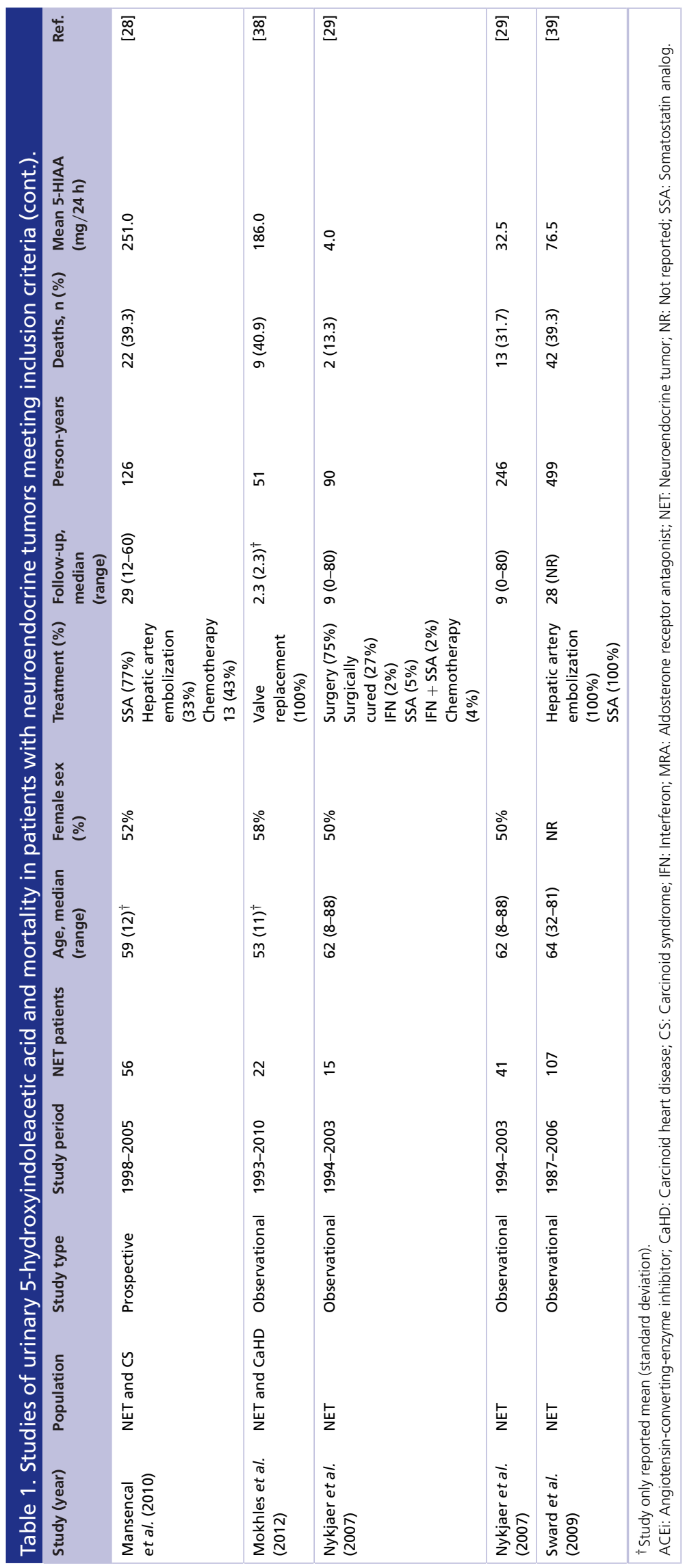




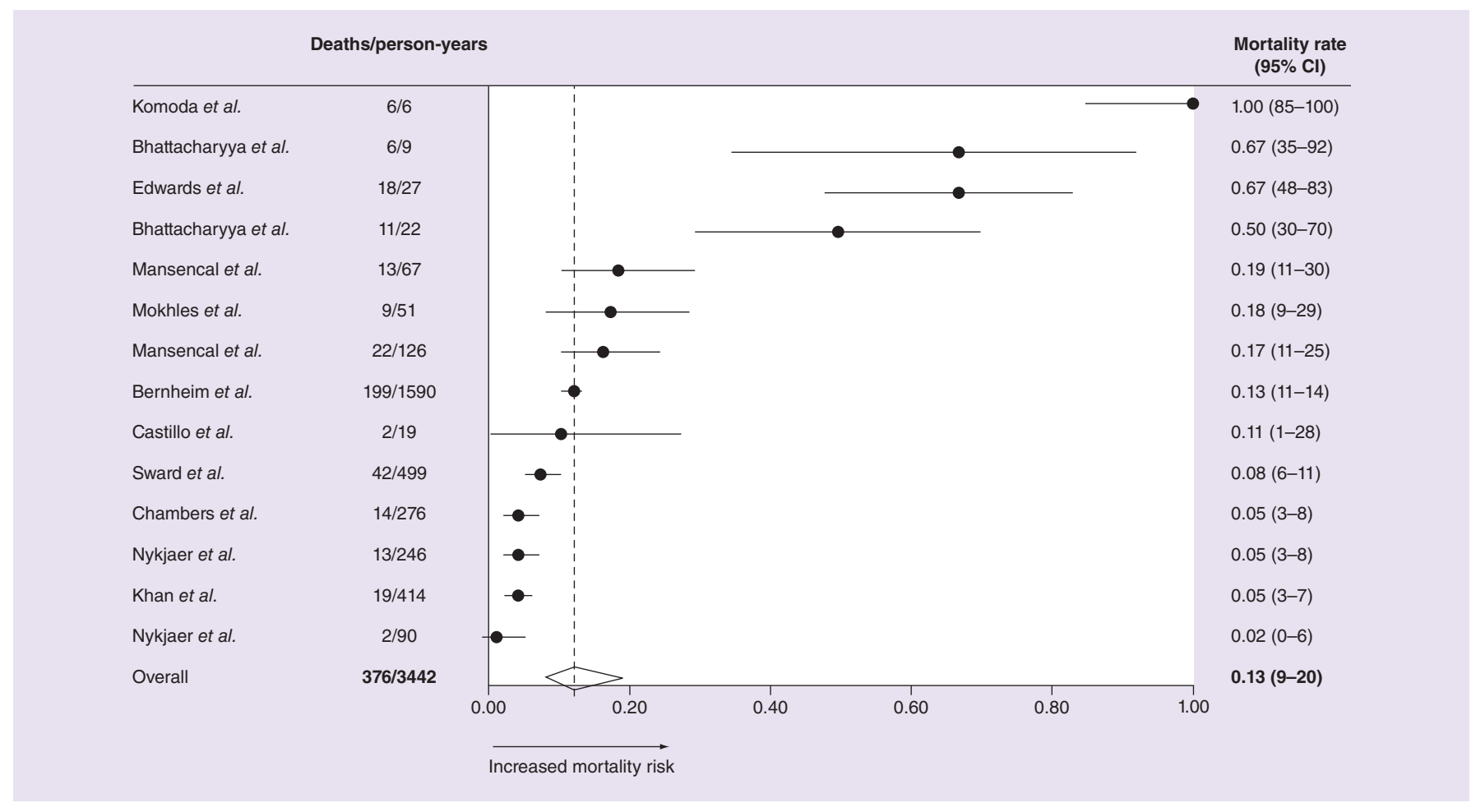

Figure 2. Forest plot of 1-year mortality in patients with neuroendocrine tumors.

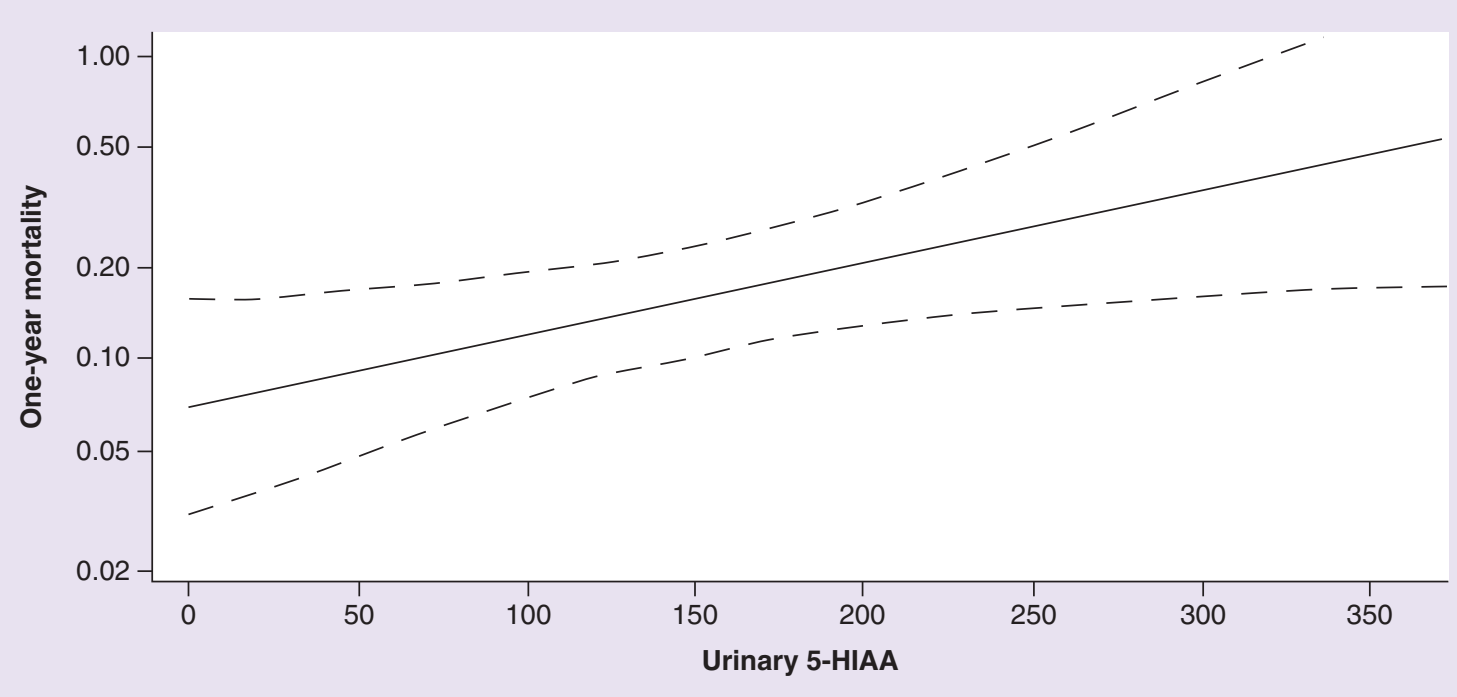

Figure 3. Meta-regression of urinary 5-hydroxyindoleacetic acid and 1-year mortality.

contribute to the cardiac manifestations [12,17,43,44]. Growing evidence suggests that serotonin (5-HT) plays a key role in the pathogenesis of $\mathrm{CaHD}$ and has been implicated in tumor growth $[18,45,46]$. Medications with serotonergic actions on human tissue have been found to lead to heart valve pathology similar to that seen in patients with NETs and CaHD [47,48]. Indeed, 5-HT has been shown to increase synthesis of TGF- $\beta$ which accumulates on heart valves $[26,49,50]$. 5-HT is metabolized by the liver and excreted in the urine as 5-HIAA which has been shown to be a reliable predictor of NET burden and CS, and can identify CaHD patients with very high sensitivity, though low specificity [17,51]. This systematic review and meta-analysis has elucidated the association between higher 5-HIAA 
and mortality even after controlling for the presence of CS or CaHD. Interestingly, mortality rates were observed to increase in the presence of CS and CaHD. A recent pooled analysis of two prospective trials in patients with NETs reported elevated 5-HIAA levels even in the absence of CS symptoms, and a significant correlation between decreases in 5-HIAA and increases in progression-free survival [52]. These findings underscore the need for effective medical management options for patients with elevated serotonin levels, especially those who have already developed CaHD, where serotonin-reducing treatments may play an important role.

This analysis should be interpreted in light of certain strengths and limitations. A broad literature review was conducted to identify the greatest number of potentially relevant studies with clearly defined inclusion parameters to investigate 24-h u5-HIAA and mortality, which yielded a considerable sample size for quantitative analysis. The search was confined to the most recent 10 years to preserve as much relevance to current clinical practice and patient populations as possible. This may have limited the number of studies reporting u5-HIAA and mortality in this population. The precision estimates around nearly all study arms, with the exception of two, independently suggested increased risk of mortality with higher levels of 5-HIAA. The estimate of heterogeneity among contributing studies was expectedly high $\left(I^{2}=93 \%\right)$ considering these were predominantly observational studies conducted in various settings worldwide and over many years.

\section{Conclusion}

This study suggests that u5-HIAA levels are predictive of 1-year mortality in patients with NETs. Further research is needed to clarify and address serotonin levels in patients with NETs, with the ultimate goal of reducing mortality in this vulnerable population.

\section{Future perspective}

To our knowledge, this meta-analysis provides the first report of a quantifiable relationship between systemic serotonin level and risk of 1-year all-cause mortality in patients with NETs. Previous reports have associated elevated serotonin with CaHD and poor outcomes, but not in a systematic manner. We report a predictable risk of 1-year mortality according to 5-HIAA levels that was further elevated in the presence of CS or CaHD. The results of our meta-analysis suggest a clear, quantifiable relationship between elevated serotonin and mortality in patients with NETs. Additional research may further clarify this relationship, including among patient subgroups and support the efforts to improve long-term outcomes for these patients.

\section{Executive summary}

- Serotonin and the burden of carcinoid heart disease (CaHD). High systemic serotonin has been implicated in cardiovascular morbidity among patients with neuroendocrine tumors, particularly those with carcinoid syndrome and $\mathrm{CaHD}$, but the relationship between mortality and serotonin in these patients has remained elusive.

- Objective of the systematic review. This systematic review and meta-analysis included studies published over the past 10 years reporting 24-h urinary levels of the serotonin metabolite 5-hydroxyindoleacetic acid (5-HIAA) and mortality in patients with neuroendocrine tumors.

- Published studies on this topic. The 14 study arms from 12 eligible studies included 755 patients (3442 person-years) and 375 deaths.

- Serotonin and mortality finding. Every ten-unit increase in $24-\mathrm{h}$ urinary 5 -HIAA predicted an $11.8 \%(95 \% \mathrm{Cl}$ : $8.9-17.0 \% ; l^{2}=93.0 \%$ ) increase in 1-year mortality.

\section{Supplementary data}

To view the supplementary data that accompany this paper please visit the journal website at: www.futuremedicine.com/doi/sup $\mathrm{pl} / 10.2217 /$ fon-2018-0960

\section{Authors' contributions}

J Zacks, VN Joish, S Shah, JC Tierce, D Patel, C McKee and P Lapuerta designed the study. J Verma (independent reviewer) and D Patel screened retrieved records. JC Tierce oversaw the statistical analysis. 


\section{Acknowledgements}

The authors would like to thank J Verma and HD Shao for their support with systematic review screening and methodological and statistical consultation. Also, HD Shao adjudicated discrepancies among screeners and provided methodological and statistical support. J Frimpter provided support in the development of this manuscript, funded by Lexicon Pharmaceuticals, Inc., and wrote the first draft of the paper and provided editorial support throughout development with input from all authors.

\section{Financial \& competing interests disclosure}

This work was funded by Lexicon Pharmaceuticals, Inc. J Zacks reports personal fees (research funding and consulting fees) from Lexicon Pharmaceuticals, Inc. VN Joish and P Lapuerta report personal fees (employment and stock ownership) from Lexicon Pharmaceuticals, Inc. S Shah, JC Tierce and D Patel report personal fees (research consulting fees) from Lexicon Pharmaceuticals, Inc. C McKee reports personal fees (employment at the time of the study) from Lexicon Pharmaceuticals, Inc. The authors have no other relevant affiliations or financial involvement with any organization or entity with a financial interest in or financial conflict with the subject matter or materials discussed in the manuscript apart from those disclosed.

Writing assistance was utilized in the production of this manuscript.

\section{Open access}

This work is licensed under the Attribution-NonCommercial-NoDerivatives 4.0 Unported License. To view a copy of this license, visit http://creativecommons.org/licenses/by-nc-nd/4.0/

\section{References}

1. Dasari A, Shen C, Halperin D et al. Trends in the incidence, prevalence, and survival outcomes in patients with neuroendocrine tumors in the United States. JAMA Oncol. 3(10), 1335-1342 (2017).

2. Garcia-Carbonero R, Capdevila J, Crespo-Herrero G et al. Incidence, patterns of care and prognostic factors for outcome of gastroenteropancreatic neuroendocrine tumors (GEP- NETs): results from the National Cancer Registry of Spain (RGETNE). Ann. Oncol. 21, 1794-1803 (2010).

3. Mocellin S, Nitti D. Gastrointestinal carcinoid: epidemiological and survival evidence from a large population-based study $(\mathrm{n}=25531)$. Ann. Oncol. 24, 3040-3044 (2013).

4. Oberg K. Neuroendocrine tumors of the digestive tract: impact of new classifications and new agents on therapeutic approaches. Curr. Opin. Oncol. 24(4), 433-440 (2012).

5. Fraenkel M, Kim M, Faggiano A et al. Incidence of gastroenteropancreatic neuroendocrine tumors: a systematic review of the literature. Endocr. Relat. Cancer 21, R153-R163 (2014).

6. Klimstra DS, Modlin IR, Coppola D, Lloyd RV, Suster S. The pathologic classification of neuroendocrine tumors: a review of nomenclature, grading, and staging systems. Pancreas 39(6), 707-712 (2010).

7. Hallet J, Law CHL, Cukier M et al. Exploring the rising incidence of neuroendocrine tumors: a population-based analysis of epidemiology, metastatic presentation, and outcomes. Cancer 121, 589-597 (2012).

8. Lewis MA, Hobday TJ. Treatment of neuroendocrine tumor liver metastases. Int. J. Hepatol. doi:10.1155/2012/973946 (2012).

9. Pandit S, Bhusal K. Carcinoid syndrome. StatPearls Publishing: Treasure Island, FL, USA. (2017) www.ncbi.nlm.nih.gov/books/NBK448096/

10. McCormick D. Carcinoid tumors and syndrome. Gastroenterol. Nurs. 25(3), 105-111 (2002).

11. Modlin IM, Sandor A. An analysis of 8305 cases of carcinoid tumors. Cancer 79, 813-829 (1997).

12. Grozinsky-Glasberg S, Grossman AB, Gross DJ. Carcinoid heart disease: from pathophysiology to treatment - "something in the way it moves". Neuroendocrinology 101(4), 263-273 (2015).

13. Lundin L, Norheim I, Landelius J, Oberg K, Theodorsson-Norheim E. Relationship of circulating vasoactive substances to ultrasound detectable cardiac abnormalities. Circulation 77(2), 264-269 (1988).

14. Pellika PA, Tajik AJ, Khandheria BK et al. Carcinoid heart disease: clinical and echocardiographic spectrum in 74 patients. Circulation 87, 1188-1196 (1993).

15. Patel C, Mathur M, Escarcega RO, Bove AA. Carcinoid heart disease: current understanding and future directions. Am. Heart J. 167(6), 789-795 (2014).

16. Ovcinnikova O, Marshall M, Bagnati F, Hoffmanns P, Taveau C, Nayroles G. A systematic literature review on carcinoid heart disease in patients with carcinoid syndrome. Poster presented at: ISPOR 22nd Annual International Meeting. Boston, MA, USA, May 2017.

17. Bhattacharyya S, Davar J, Dreyfus G, Caplin ME. Carcinoid heart disease. Circulation 116, 2860-2865 (2007).

18. Møller JE, Pellikka PA, Bernheim AM, Schaff HV, Rubin J, Connolly HM. Prognosis of carcinoid heart disease: analysis of 200 cases over two decades. Circulation 112(21), 3320-3327 (2005). 
19. Zandee WT, Kamp K, van Adrichem RC, Feelders RA, de Herder WW. Limited value for urinary 5-HIAA excretion as prognostic marker in gastrointestinal neuroendocrine tumors. Eur. J. Endocrinol. 175(5), 361-366 (2016).

20. Tirosh A, Nilubol N, Patel D, Kebebew E. Prognostic utility of 24 hour urinary 5-HIAA doubling time in patients with neuroendocrine tumors. Endocr. Pract. 24(8), 710-717 (2018).

21. Cochrane Handbook for Systematic Reviews of Interventions. Higgins JPT, Green S (Eds).Version 5.1.0 (updated March 2011). The Cochrane Collaboration (2011). www.handbook.cochrane.org

22. DerSimonian R, Laird N. Meta-analysis in clinical trials. Control. Clin. Trials. 7(3), 177-188 (1986).

23. Viechtbauer W. Conducting meta-analyses in R with the metaphor package. J. Stat. Softw. 36(3), 1-48 (2010).

24. Schwarzer G. Meta: an R package for meta-analysis. R News 7(3), 40-45 (2007).

25. Pasek J. Weights: weighting and weighted statistics. (2018). https://CRAN.R-project.org/package=weights

26. Möbius TWD. Metagen: inference in meta analysis and meta regression. (2014). https://CRAN.R-project.org/package=metagen

27. Sievert C, Parmer C, Hocking T, Chamberlain S, Ram K, Corvellec M, Despouy P. Plotly: create interactive web graphics via 'plotly.js'. (2017). https://cran.R-project.org/package=plotly

28. Mansencal N, McKenna WJ, Mitry E et al. Comparison of prognostic value of tissue Doppler imaging in carcinoid heart disease versus the value in patients with the carcinoid syndrome but without carcinoid heart disease. Am. J. Cardiol. 105(4), 527-531 (2010).

29. Nykjaer KM, Gronbaek H, Nielsen DT, Christiansen P, Astrup LB. Description of patients with midgut carcinoid tumors: clinical database from a Danish centre. In Vivo 21, 679-84 (2007).

30. Bernheim AM, Connolly HM, Rubin J et al. Role of hepatic resection for patients with carcinoid heart disease. Mayo Clinic Proc. 83(2), $143-150$ (2008).

31. Bhattacharyya S, Raja SG, Toumpanakis C, Caplin ME, Dreyfus GD, Davar J. Outcomes, risks and complications of cardiac surgery for carcinoid heart disease. Eur. J. Cardiothorac. Surg. 40(1), 168-172 (2011).

32. Bhattacharyya S, Jagroop A, Gujral DM et al. Circulating plasma and platelet 5- hydroxytryptamine in carcinoid heart disease: a pilot study. J. Heart Valve Dis. 22, 400-407 (2013).

33. Castillo J, Filsoufi F, Adams D, Raikhelkar J, Zaku B, Fischer G. Management of patients undergoing multivalvular surgery for carcinoid heart disease: the role of the anaesthetist. Br. J. Anaesth. 101(5), 618-626 (2008).

34. Chambers AJ, Pasieka JL, Dixon E, Rorstad O. The palliative benefit of aggressive surgical intervention for both hepatic and mesenteric metastases from neuroendocrine tumors. Surgery 144(4), 645-53 (2008).

35. Edwards NC, Yuan M, Nolan O et al. Effect of valvular surgery in carcinoid heart disease: an observational cohort study. J. Clin. Endocrinol. Metab. 101(1), 183-190 (2016).

36. Khan MS, El-Khouly F, Davies P, Toumpanakis C, Caplin ME. Long-term results of treatment of malignant carcinoid syndrome with prolonged release lanreotide (Somatuline Autogel). Aliment Pharmacol. Ther. 34(2), 235-242 (2011).

37. Komoda S, Komoda T, Pavel ME et al. Cardiac surgery for carcinoid heart disease in 12 cases. Gen. Thorac. Cardiovasc. Surg. 59(12), 780-785 (2011).

38. Mokhles P, Herwerden LAV, Jong PLD et al. Carcinoid heart disease: outcomes after surgical valve replacement. Eur. J. Cardiothorac. Surg. 41(6), 1278-1283 (2011).

39. Swärd C, Johanson V, Dijkum ENV et al. Prolonged survival after hepatic artery embolization in patients with midgut carcinoid syndrome. Br. J. Surg. 96(5), 517-521 (2009).

40. Maroun J, Kocha W, Kvols L et al. Guidelines for the diagnosis and management of carcinoid tumors. Part 1: the gastrointestinal tract. a statement from a Canadian national carcinoid expert group. Curr. Oncol. 13(2), 67-76 (2006).

41. Gustafsson BI, Hauso O, Drozdov I, Kidd M, Modlin IM. Carcinoid heart disease. Int. J. Cardiol. 129(3), 318-324 (2008).

42. Zuetenhorst JM, Bonfrer JMGM, Korse CM, Bakker R, van Tinteren H, Taal BG. The role of urinary 5-hydroxyindoleacetic acid excretion and plasma levels of atrial natriuretic peptide, transforming growth factor-beta and fibroblast growth factor. Cancer 97 , 1609-1615 (2003).

43. Davar J, Connolly HM, Caplin ME et al. Diagnosing and managing carcinoid heart disease in patients with neuroendocrine tumors: an expert statement. J. Am. Coll. Cardiol. 69(10), 1288-1304 (2017).

44. Fox DJ, Khattar RS. Carcinoid heart disease: presentation, diagnosis, and management. Heart 90(10), 1224-1228 (2004).

45. Denney WD, Kemp WE, Anthony LB, Oates JA, Byrd BF. Echocardiographic and biochemical evaluation of the development and progression of carcinoid heart disease. J. Am. Coll. Cardiol. 32(4), 1017-1022 (1998).

46. Siddiqui EJ, Thompson CS, Mikhailidas DP, Mumta FH. The role of serotonin in tumor growth. Oncol. Rep. 14(6), 1593-1597 (2005).

47. Feldman JM. Carcinoid tumors and syndrome. Semin. Oncol. 14(3), 237-246 (1987).

48. Dahl CF, Allen MR, Urie PM, Hopkins PN. Valvular regurgitation and surgery associated with fenfluramine use: an analysis of 5743 individuals. BMC Med. 6, 34 (2008). 
49. Connolly H, Crary J, McGoon M et al. Valvular heart disease associated with fenfluraminephentermine. N. Engl. J. Med. 337, 581-588 (1997).

50. Jagroop I, Mikhailidis D. An investigation of the serotonergic effects of fenfluramine, dexfenfluramine and dexnorfenfluramine using platelets as neuronal models. Platelets 11, 161-165 (2000).

51. Kulke MH, Shah MH, Benson AB et al. Neuroendocrine tumors, version 1.2015. J. Natl Compr. Canc. Netw. 13(1), 78-108 (2015).

52. Mirakhur B, Pavel ME, Pommier RF et al. Biochemical responses in symptomatic and asymptomatic patients with neuroendocrine tumors: pooled analysis of two Phase 3 trials. Endocr. Pract. 24, 948-962 (2018). 